\title{
Boundaries of Smooth Strictly Convex Sets in the Euclidean Plane $R^{2}$
}

\author{
Horst Kramer \\ Retired, Niedernhausen, Germany \\ Email:f.h.kramer@gmail.com
}

How to cite this paper: Kramer, H. (2017) Boundaries of Smooth Strictly Convex Sets in the Euclidean Plane $\mathrm{R}^{2}$. Open Journal of Discrete Mathematics, 7, 71-76.

https://doi.org/10.4236/ojdm.2017.72008

Received: February 24, 2017

Accepted: April 16, 2017

Published: April 19, 2017

Copyright $\odot 2017$ by author and Scientific Research Publishing Inc. This work is licensed under the Creative Commons Attribution International License (CC BY 4.0).

http://creativecommons.org/licenses/by/4.0/ (c) (i)

Open Access

\begin{abstract}
We give a characterization of the boundaries of smooth strictly convex sets in the Euclidean plane $R^{2}$ based on the existence and uniqueness of inscribed triangles.
\end{abstract}

\section{Keywords}

Strict Convexity, Smoothness, Supporting Lines, Inscribed Triangles

\section{Introduction}

The reader unfamiliar with the theory of convex sets is referred to the books [1] [2] [3] [4] [5]. Let $M$ be a set in the $n$-dimensional Euclidean space $R^{n}$. In the following we shall denote by int $M, c l M, \vartheta M, \operatorname{conv} M$ the interior, the closure, the boundary and respectively the convex hull of the set $M$. With $d(x, y)$ we denote the Euclidean distance of the points $x$ and $y$ and with $L(x, y)$ the line determined by the points $x$ and $y$. The diameter diam $M$ of a set $M$ is $\operatorname{diam} M=\sup \{d(x, y): x, y \in M\}$. For a point $p \in R^{2}$ and a real number $r$ we shall denote with $C(p, r)$ and $D(p, r)$ the circle and respectively the disc with center $p$ and radius $r$. The distance $d(p, M)$ between a point $p$ and a set $M$ in $R^{2}$ is $d(p, M)=\inf \{d(p, x): x \in M\}$. With $] x, y[$ we denote the open line segment with endpoints $x$ and $y$, that is ]$x, y\left[=\operatorname{conv}\{x, y\} \backslash\{x, y\}\right.$. For 3 nonlinear points $x, y$ and $z$ in $R^{2}$ we denote with $\max \angle(x, y, z)$ the maximum angle of the triangle $\Delta(x, y, z)$. A convex curve is a connected subset of the boundary of a convex set.

\section{Preliminaries}

In the chapter 8 of the book [4] of F.A. Valentine the author says the following:

"It is interesting to see what kind of strong conclusions can be obtained from 
weak suppositions about any triplet of points of a plane set $S$." In [6] Menger gives such a characterization of the boundary of a convex plane set $S$ based on intersection properties of $S$ with the seven convex sets in which the space $R^{2}$ is subdivided by the lines $L\left(x_{1}, x_{2}\right), L\left(x_{2}, x_{3}\right)$ and $L\left(x_{3}, x_{1}\right)$ determined by an arbitrary triplet of noncollinear points $\left\{x_{1}, x_{2}, x_{3}\right\}$ from $S$. In [7] K. Juul proved the following:

Theorem 1. A plane set $S$ fulfils

1) $\forall x, y, z \in S: S \cap \operatorname{int}\{\operatorname{conv}\{x, y, z\}\}=\varnothing$, if and only if $S$ is either a subset of the boundary of a convex set, or an $X$-set, that is a set $\left\{x_{1}, x_{2}, x_{3}, x_{4}, x_{5}\right\}$ with $] x_{1}, x_{2}[\cap] x_{3}, x_{4}\left[=\left\{x_{5}\right\}\right.$.

A survey of different characterizations of convex sets is given in the paper [8]. The results of K. Menger and that of K. Juul give characterizations of the boundaries of convex sets.

In the years 1978 [9] and 1979 [10] we have proved the following two theorems giving a characterization of the boundaries of smooth strictly convex sets:

Theorem 2. A plane compact set $S$ is the boundary of a smooth strictly convex set if and only if the following two conditions hold:

1) $\forall x, y, z \in S: S \cap \operatorname{int}\{\operatorname{conv}\{x, y, z\}\}=\varnothing$,

2) For every triangle $\Delta\left(p_{1}, p_{2}, p_{3}\right)$ in $R^{2}$ there is one and only one triangle $\Delta\left(p_{1}^{\prime}, p_{2}^{\prime}, p_{3}^{\prime}\right)$ homothetic to the triangle $\Delta\left(p_{1}, p_{2}, p_{3}\right)$ inscribed in the set $S$, i.e. such that $p_{1}^{\prime}, p_{2}^{\prime}, p_{3}^{\prime} \in S$.

Theorem 3. A plane compact set $S$ is the boundary of a smooth strictly convex set if and only if the following two conditions hold:

1) For every $p \in S$ and every $\epsilon>0$ there is a positive number $\delta(p, \epsilon)$ such that for every triplet of nonlinear points $r, s, t$ in $S \cap \operatorname{int}\{D(p, \delta(p, \epsilon))\}$ we have $\max \angle(r, s, t)>\pi-\epsilon$.

2) For every triangle $\Delta\left(p_{1}, p_{2}, p_{3}\right)$ in $R^{2}$ there is one and only one triangle $\Delta\left(p_{1}^{\prime}, p_{2}^{\prime}, p_{3}^{\prime}\right)$, homothetic to the triangle $\Delta\left(p_{1}, p_{2}, p_{3}\right)$ inscribed in the set $S$, i.e. such that $p_{1}^{\prime}, p_{2}^{\prime}, p_{3}^{\prime} \in S$.

\section{Main Results}

The main result of this paper is Theorem 4 giving another characterization of the boundaries of smooth strictly convex sets in the Euclidean plane $R^{2}$ which uses also condition (2) of the Theorem 2 and Theorem 3.

Theorem 4. A compact set $S$ in the Euclidean plane $R^{2}$ is the boundary of a smooth strictly convex set if and only if there are verified the following three conditions:

1) For every triangle $\Delta\left(p_{1}, p_{2}, p_{3}\right)$ in $R^{2}$ there is one and only one triangle $\Delta\left(p_{1}^{\prime}, p_{2}^{\prime}, p_{3}^{\prime}\right)$ homothetic to the triangle $\Delta\left(p_{1}, p_{2}, p_{3}\right)$ inscribed in the set $S$, i.e. such that $p_{1}^{\prime}, p_{2}^{\prime}, p_{3}^{\prime} \in S$.

2) For any two distinct points $p \in S$ and $q \in S$ there are at least two points $t_{1}$ and $t_{2}$ such that $t_{1} \in S \cap H_{1}$ and $t_{2} \in S \cap H_{2}$, where $H_{1}$ and $H_{2}$ are the two open halfplanes generated in $R^{2}$ by the line $L(p, q)$.

3) The set $S$ does not contain three collinear points. 
For the proof of Theorem 4 we need the following theorem from the paper [11] and three lemmas:

Theorem 5. Let $\Delta(a, b, c)$ be a triangle in the Euclidean plane $R^{2}$. Suppose that $S$ is a strictly convex closed arc of class $C^{1}$. Then there exists a single triangle $\Delta\left(a_{1}, b_{1}, c_{1}\right)$ homothetic to the triangle $\Delta(a, b, c)$ inscribed in the set $S$, in the sense that $a_{1}, b_{1}, c_{1} \in S$.

Lemma 1. The convex hull convS of a compact set $S$ in the Euclidean plane $R^{2}$ verifying the condition (2) from Theorem 4 is a strictly convex set.

Proof. Let us suppose the contrary. Then there are two distinct points $p, q \in \vartheta\{\operatorname{convS}\}$ such that the line segment $\operatorname{conv}\{p, q\} \subset \operatorname{conv} S$. The convex hull of a compact set is also a compact set (see [5] Theorem 2.2.6). The line $L(p, q)$ is thereby a supporting line for the compact set convS. Denote with $H_{1}$ and $H_{2}$ the two open halfplanes generated by the line $L(p, q)$ such that convS $\subset \mathrm{cl}\left\{H_{1}\right\}$ and $H_{2} \cap \operatorname{conv} S=\varnothing$. By Carathodory's Theorem (see [5] or [12] Theorem 2.2.4) the point $p \in$ convS can be expressed as a convex combination of 3 or fewer points of $S$.

If the point $p$ can be expressed only as a convex combination of three (and not of fewer) points $x_{1}, x_{2}, x_{3}$ of $S$ then it follows that we must have $p \in \operatorname{int}\left\{\operatorname{conv}\left\{x_{1}, x_{2}, x_{3}\right\}\right\} \subset \operatorname{int}\{\operatorname{conv} S\}$ in contradiction to the fact that $p \in \vartheta\{\operatorname{conv} S\}$.

If the point $p$ can be expressed only as a convex combination of 2 (and not of fewer) points of $S$, there are $x_{1} \in S$ and $x_{2} \in S$ such that $p \in \operatorname{conv}\left\{x_{1}, x_{2}\right\} \subset \operatorname{conv} S \subset c l\left\{H_{1}\right\}$. Then the points $x_{1}$ and $x_{2}$ must be on the supporting line $L(p, q)$. As $H_{2} \cap \operatorname{conv} S=\varnothing$, this is in contradiction with property (2) of the set $S$.

Thereby we must have $p \in S$. By an analog reasoning for the point $q$ we can conclude that we have also: $q \in S$. Thus we have proved the existence of at least 2 different points of $S$ on the supporting line $L(p, q)$ of convS in contradiction to the property (2) of the set $S$.

Lemma 2. The boundary $\vartheta\{$ convS $\}$ of the convex hull of a compact set $S$ in the Euclidean plane $R^{2}$ verifying the condition (2) from Theorem 4 is a subset of the set $S$, i.e. $\vartheta\{\operatorname{conv} S\} \subset S$.

Proof. Let $p \in \vartheta\{$ convS\} be an arbitrary point from the boundary of the convex hull of the compact set $S$. Each boundary point of the compact convex set convS in $R^{2}$ is situated on at least one supporting line of the set convS (see for instance [3] pp. 6). We distinguish now the following two cases:

1) There is only one supporting line $L_{1}$ of the set convS going through the point $p$, i.e. the boundary $\vartheta\{$ convS\} is smooth in the point $p$. By Lemma 1 it follows that the convex hull convS is a strictly convex set and thereby we have conv $S \cap L_{1}=p$.

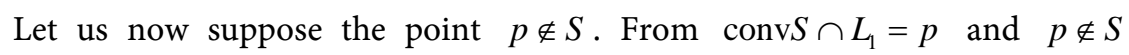
follows then $S \cap L_{1}=\varnothing$. Denote with $H_{o}$ the open halfplane generated by the line $L_{1}$, which contains the set $S$. As $S$ is a compact set we have then $r=\min \left\{d\left(x, L_{1}\right): x \in S\right\}>0$. Consider then in the open halfplane $H_{o}$ a line 
$L_{1}^{\prime}$ parallel to the line $L_{1}$ at distance $r$ to the line $L_{1}$. Denote with $H_{o}^{\prime}$ the open halfplane generated by the line $L_{1}^{\prime}$ and such that $H_{o}^{\prime} \subset H_{o}$. It is evident that $p \notin c l\left\{H_{o}^{\prime}\right\}$. From the definition of the constant, $r$ folows $S \subset \operatorname{cl}\left\{H_{o}^{\prime}\right\}$ and $\vartheta\{\operatorname{convS}\} \subset c l\left\{H_{o}^{\prime}\right\}$ in contradiction to $p \in \vartheta\{\operatorname{convS}\}$. Thereby our supposition $p \notin S$ is false, i.e. we must have $p \in S$.

2) There are two supporting lines $L_{1}$ and $L_{2}$ of the set convS going through the point $p$. Denote then with $L_{1}^{\prime}$ and $L_{2}^{\prime}$ the two halflines with endpoint $p$ of the line $L_{1}$ and respectively $L_{2}$ such that $\operatorname{conv} S \subset \operatorname{conv}\left\{L_{1}^{\prime} \cup L_{2}^{\prime}\right\}$.

Let us suppose that $p \notin S$. From the compactness of $S$ follows then the existence of a real number $r>0$ such that for the disc $D(p, r)$ with the center $p$ and the radius $r$ we have: $D(p, r) \cap S=\varnothing$. Consider then the points $q_{1}=C(p, r) \cap L_{1}^{\prime}$ and $q_{2}=C(p, r) \cap L_{2}^{\prime}$, where $C(p, r)$ is the circle with center $p$ and radius $r$. Let $H_{1}$ be the open halfplane generated by the line $L\left(q_{1}, q_{2}\right)$, which contains the point $p$ and $H_{2}$ the other open halfplane generated by the line $L\left(q_{1}, q_{2}\right)$. We have then evidently $S \cap c l H_{1}=\varnothing$ and thereby $S \subset H_{2}$. From the inclusion $S \subset H_{2}$ it follows also that convS $\subset H_{2}$. As $\vartheta\{\operatorname{conv} S\} \subset S$ we have also: $\vartheta\{\operatorname{conv} S\} \subset H_{2}$ in contradiction to our supposition $p \in \vartheta\{\operatorname{conv} S\}$. Therefore the point $p$ must belong to the set $S$.

So we have proved in both cases (1) and (2) that $p \in \vartheta\{\operatorname{convS}\}$ implies $p \in S$, i.e. $\vartheta\{\operatorname{conv} S\} \subset S$.

A characterization of compact sets $S$ in the Euclidean plane $R^{2}$ for which we have $S=\vartheta\{$ convS $\}$ is given in the following:

Lemma 3. A compact set $S$ in the Euclidean plane $R^{2}$ has a strictly convex hull and coincides with the boundary of its convex hull $\vartheta\{\operatorname{conv} S\}$ if and only if there are verified the conditions (2) and (3).

Proof. Let $S$ be a compact set in the Euclidean plane $R^{2}$, which has a strictly convex hull convS and such that $S=\vartheta\{\operatorname{conv} S\}$. Consider then two arbitrary points $p_{1}$ and $p_{2}$ of the set $S$ and the two open halfplanes generated by the line $L\left(p_{1}, p_{2}\right)$ in $R^{2}$. Because $S$ has a strictly convex hull it is then evident that we have verified condition (2) and (3).

To prove the only if part of the lemma let us consider a compact set $S$ in the Euclidean plane $R^{2}$, which verifies conditions (2) and (3). By Lemma 1 the convex hull convS of $S$ is a strictly convex set. By Lemma 2 we have then for the set $S$ the inclusion $\vartheta\{\operatorname{conv} S\} \subset S$. Let us now suppose that we would have $S \not \subset \vartheta\{\operatorname{conv} S\}$, i.e. there is a point $p \in S$ such that $p \notin \vartheta\{\operatorname{conv} S\}$. Then the point $p$ must be an interior point of the convex hull convS. Let $L$ be an arbitrary line such that $p \in L$. Then it is obvious that the line $L$ intersects $\vartheta\{$ conv $S\}$ in two different points $t_{1}$ and $t_{2}$ such that $p \in \operatorname{conv}\left\{t_{1}, t_{2}\right\}$. From $\vartheta\{\operatorname{conv} S\} \subset S$ it follows that $t_{1} \in S$ and $t_{2} \in S$ in contradiction to the condition (3) of the set $S$. So we conclude that $S \subset \vartheta\{\operatorname{conv} S\}$. This inclusion together with the inclusion $\vartheta\{$ conv $S\} \subset S$ gives then $S \subset \vartheta\{$ conv $S\}$.

A similar result as that of Lemma 3 without the compactness of the set $S$ but with the additional assumption of the connectedness of the set $S$ was obtained 
by K. Juul in [7]:

Theorem 6. A connected set $S$ in $R^{2}$ is a convex curve if and only if it verifies condition (1) from Theorem 1.

\section{Proof of Theorem 4.}

For the proof of the if-part of the theorem let $S$ be the boundary of a compact smooth strictly convex set in the Euclidean plane $R^{2}$. It is then easy to verify conditions (2) and (3) for the set $S$. Condition (1) follows immediately from Theorem 5.

For the proof of the "only if"- part of the theorem let $S$ be a compact set in the Euclidean plane $R^{2}$, which verifies conditions (1), (2) and (3). By Lemma 3 it follows that the convex hull convS of the set $S$ is strictly convex and that $S=\vartheta\{\operatorname{conv} S\}$.

It remains to prove that convS is also a smooth set. Let us assume the contrary: there is a point $a_{1} \in \mathcal{Y}\{\operatorname{conv} S\}$, which is not a smooth point of the boundary of $S$, i.e. there exist two supporting lines $L_{1}$ and $L_{2}$ for the set convS at the point $a_{1}$. For $i \in\{1,2\}$ denote with $H_{i}$ the closed half-plane generated by the supporting line $L_{i}$, which contains the set $S$. Denote with $C$ the convex cone $C=H_{1} \cap H_{2}$. We have then evidently the inclusions: $S \subset C$ and conv $S \subset C$. As convS is a strictly convex set we have also the inclusion $S \backslash a_{1} \subset \operatorname{int} C$. For $i \in\{1,2\}$ denote with $L_{i}^{\prime}$ the closed halfline of the line $L_{i}$ with origin $a_{1}$ such that $L_{i}^{\prime} \subset \vartheta C$. Consider then the isosceles triangle $\Delta\left(a_{1}, a_{2}, a_{3}\right)$ with $d\left(a_{1}, a_{2}\right)=d\left(a_{1}, a_{3}\right)$ and such that angle $\angle a_{2} a_{1} a_{3}$ has the same angle bisector as the boundary angle of the cone $C$ formed by the halflines $L_{1}^{\prime}$ and $L_{2}^{\prime}$ with the vertex $a_{1}$ and such that the angle $\angle a_{2} a_{1} a_{3}$ is greater than the boundary angle of the cone $C$. By condition (1) there exists then three points $a_{i}^{\prime} \in S, i=1,2,3$ such that triangle $\Delta\left(a_{1}^{\prime}, a_{2}^{\prime}, a_{3}^{\prime}\right)$ is homothetic to the triangle $\Delta\left(a_{1}, a_{2}, a_{3}\right)$. Because the angle $\angle a_{2} a_{1} a_{3}$ is greater than the boundary angle of the cone $C$ the point $a_{1}^{\prime}$ cannot coincide with the point $a_{1}$. From this fact and the inclusion $S \backslash a_{1} \subset \operatorname{int} C$ we can conclude that we have: $a_{i}^{\prime} \in \operatorname{int} C$ for $i=1,2,3$. From the homothety of the triangles $\Delta\left(a_{1}^{\prime}, a_{2}^{\prime}, a_{3}^{\prime}\right)$ and $\Delta\left(a_{1}, a_{2}, a_{3}\right)$ it follows then that $a_{1}^{\prime} \in \operatorname{int}\left\{\operatorname{conv}\left\{a_{1}, a_{2}^{\prime}, a_{3}^{\prime}\right\}\right\} \subset \operatorname{int}\{\operatorname{convS}\}$ in contradiction to $a_{1}^{\prime} \in S=\vartheta\{\operatorname{conv} S\}$. So we have proved that the convex hull convS is a smooth strictly convex set.

\section{Conclusions}

As we have seen condition (1) is used and is essential in the proofs of the Theorem 2, Theorem 3 and Theorem 4 . We emit now the following:

Conjecture: A compact set $S$ in the Euclidean plane $R^{2}$ is the boundary of a smooth strictly convex set if and only if there is verified the condition:

For every triangle $\Delta\left(p_{1}, p_{2}, p_{3}\right)$ in $R^{2}$ there is one and only one triangle $\Delta\left(p_{1}^{\prime}, p_{2}^{\prime}, p_{3}^{\prime}\right)$ homothetic to the triangle $\Delta\left(p_{1}, p_{2}, p_{3}\right)$ and inscribed in the set $S$ i.e. such that $p_{1}^{\prime}, p_{2}^{\prime}, p_{3}^{\prime} \in S$.

P. Mani-Levitska cites in his survey [8] the papers [7] and [9] and says reffering to these, that he has not encountered extensions of these results to higher 
dimensions. We also don't know generalizations of our results to higher dimensions.

\section{Acknowledgements}

The author is grateful to the referees for the helpful comments.

\section{References}

[1] Blaschke, W. (1916) Kreis und Kugel. Verlag von Veit \& Comp, Leipzig.

[2] Boltyanski, V., Martini, H. and Soltan, P.S. (1997) Excursion into Combinatorial Geometry. Springer-Verlag, Heidelberg. https://doi.org/10.1007/978-3-642-59237-9

[3] Bonnesen, T. and Fenchel, W. (1974) Theorie der konvexen Körper. Springer-Verlag, Heidelberg. https://doi.org/10.1007/978-3-642-93014-0

[4] Valentine, F.A. (1968) Konvexe Mengen. Hochschultaschenbücher-Verlag, Mannheim.

[5] Webster, R. (1994) Convexity. Oxford University Press, Oxford.

[6] Menger, K. (1931) Some Applications of Point Set Methods. Annals of Mathematics, 32, 739-750. https://doi.org/10.2307/1968317

[7] Juul, K. (1975) Some Three-Point Subset Properties Connected with Menger's Characterization of Boundaries of Plane Convex Sets. Pacific Journal of Mathematics, 58, 511-515. https://doi.org/10.2140/pjm.1975.58.511

[8] Mani-Levitska, P. (1993) Characterization of Convex Sets. In: Gruber, P.M. and Wills, J.M., Eds., Handbook of Convex Geometry, North-Holland, 19-41.

[9] Kramer, H. (1978) A Characterization of Boundaries of Smooth Strictly Convex Plane Sets. Revue d'analyse numérique et de théorie de l'approximation, 7, 61-65.

[10] Kramer, H. (1979) Boundaries of Smooth Strictly Convex Plane Sets. Revue d'analyse numérique et de théorie de l'approximation, 8, 59-66.

[11] Kramer, H. and Németh, A.B. (1972) Triangles Inscribed in Smooth Closed Arcs. Revue d'analyse numérique et de théorie de l'approximation, 1, 63-71.

[12] Caratheodory, C. (1911) Ueber den Variabilitaetsbereich der Fourierschen Konstanten von positiven harmonischen Funktionen. Rendiconti del Circolo Matematico di Palermo, 32, 193-217. https://doi.org/10.1007/BF03014795

Submit or recommend next manuscript to SCIRP and we will provide best service for you:

Accepting pre-submission inquiries through Email, Facebook, LinkedIn, Twitter, etc. A wide selection of journals (inclusive of 9 subjects, more than 200 journals)

Providing 24-hour high-quality service

User-friendly online submission system

Fair and swift peer-review system

Efficient typesetting and proofreading procedure

Display of the result of downloads and visits, as well as the number of cited articles

Maximum dissemination of your research work

Submit your manuscript at: http://papersubmission.scirp.org/

Or contact ojdm@scirp.org 